\title{
Emotion Measurement for Virtual Systems Laboratory: Perspective of Affective Design
}

\author{
Huang Hailin \\ Institute of Higher Education, Jilin University \\ Changchun, P.R.China \\ huanghl@jlu.edu.cn
}

\author{
Wu Shuang \\ Institute of Higher Education, Jilin University \\ Changchun, P.R.China \\ azureblue@vip.qq.com
}

\begin{abstract}
The study discussed emotional experience of learners on a Virtual Systems Laboratory (VSL) setting in relation to the thoughts of Affective Design (AD), of primary concern were emotion measurement of learning perceptions. Centre to the thesis of paper is emotional keywords extractions though backward inference method that roots for pictographic pictures to measure the emotional experience. The work explores ways to jump-start deeper understanding toward learning experience for related designers and practitioners before substantial progress can be made.
\end{abstract}

Keywords-Affective Design; Virtual Systems Laboratory; Emotional Experience; Emotion Measurement

\section{INTRODUCTION}

The interest in widely adoption of virtual learning platforms [1-2] indicate Virtual Systems Laboratory (VSL), which can be used as a preliminary phase to familiar with real instrumentation, holds out great promises for the near future. However, insufficient emphasis has been placed on the learning experience induced by technological concentration in design process of VSL. This trend of design was relying on a combination of state-of-the-art technologies and suppressing the emotional nature of cognitive learning, which in turn, learners may somehow ignore much of what makes the experiment a rewarding one to undertake, and the learning outcome might be weakened eventually.

In contrast to the ample amount of the technologicalconcentrated researches, a set of prescriptive propositions obtained from affective design and measurement was presented within a framework in this paper. We contend the success of laboratory instruction hinges on designing a learning platform of distributing educational context in a way that enriched with subjective or unconscious feelings into learning experiences. So, we begin with a brief overview of $\mathrm{AD}$ and Emotional Experience, then examine how they related to emotion measurement of VSL and how emotional keywords extractions process might be conceptualized.

\section{A. Affective/Kansei Design}

The word Kansei (or affective) comes from Japanese, to clarify the inner feelings towards different objects, and improve methods capable of grasping affective values and translating them into design solutions that fit feelings of users. Prior research [3] point out that Kansei means the customer's psychological feeling as well as embracing physiological issues, it is defined as 'translating the customer's feeling into the product domain'. If the user's target is a passenger car, all emotional feelings concerning the exterior, interior, engine, etc. are implemented in those designs.

\section{B. Emotional Experience}

The other line of theoretical foundation comes from the perspectives of Psychology. The word 'experience' refers to those immediately perceived but mentally unprocessed feelings [4]. For learners who need external cues to be stimulated or emotionally affected, we may direct their awareness to limit the stimulus field by adding affective elements or values in VSL. Also, if VSL can provide specific guidance or interventions to enrich the mental experience of learning, learners with higher intrinsic emotions, who can immerse in learning environment no matter what kind of situation is designed like, are more likely to succeed. Furthermore, add 'spur-the-moment experience' that arise interest spontaneously due to environmental factors embedded in learning platform, would also increases emotional experience when an experiment or to-be-learned knowledge is novel, or relevant to educational goals.

As shown in Fig 1, affective design is based on emotional factors of VSL, as to where efforts shall be focused to design a positive emotional experience.

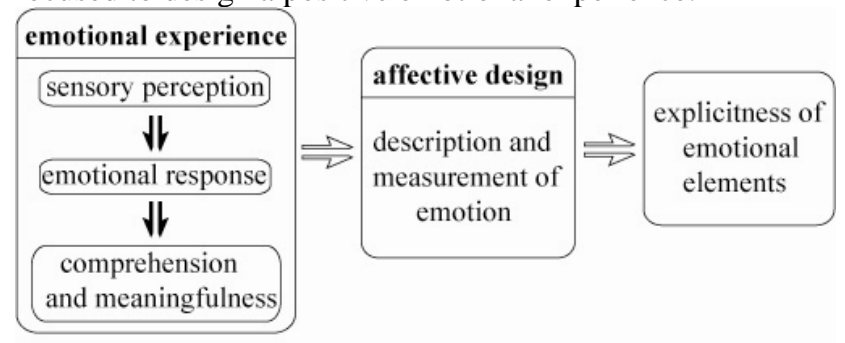

Fig. 1. Emotional experience process based on Affective Design

\section{INFLUENCE FACTOR OF EMOTIONAL MEASUREMENT}

\section{A. Emotional Need}

Emotional need of learning is the mental requirement that centres on feelings such as happy, anger and frustration [5-6], normally involves the understanding part of educators or peers. In VSL settings, designers may centre on the idea that 
experiments in the given genre tend to have amusement features as in games. When designing VSL to students at elementary schools, where learners may as frequently driven by game elements due to children often directed toward the pursuit of fantasies and colourful stuffs. Kids want to be entertained and creatively stimulated, if designers create situational interests which can dazzle senses, touch hearts, and stimulate minds, it appears to be helpful to VSL instruction.

\section{B. Emotional Cognition}

Emotional cognition is triggered by the brain's appraisal of a stimulus with respect to the perceiver's goals. In VSL settings, the function, operational interface, and other design details concerned with visual image, including light, colour, and combination of settings, should be arranged properly to help with learning by transferring knowledge from text segments.

\section{EMOTION MEASUREMENT OF VSL GROUNDED IN AFFECTIVE DESIGN}

\section{A. Emotion Description and Measurement}

Category Classification is a commonly used method using a tree structure from a main event to subsequent sub events to get affective factors and design details simultaneously. In this process, affective words are considered as dependent variables. The purpose is to find the most contributory product characteristics to satisfy the feelings of learner. In this paper, we use backward inference method to transfer affective factors into emotional evaluation elements, so as to bridge the relationships between emotional experience and VSL.

Sensory quantitative method for emotion measurement methods mainly have the physiological and psychological methods, due to physiological measurement is complex, and requires the designer to have a certain knowledge of statistics, so in actual measurement, we commonly use psychology measurement method. Therefore, Semantic Differential (SD) method was employed to examine the relationship between the perceived evaluation and form affective elements. In the vocabulary classification method, we use the five-point rating method to extract representative factors to measure scales defined with contrasting adjectives at each end.

\section{B. MEmotion Measurement of VSL}

1 ) Define Emotional Keywords. In the emotional psychology study, our foundation lies on the construct of cognitive psychology, which prior research [7] has shown its potential impact among 16 emotional dimensions, such as happiness, pleasure, impulsiveness, excitement, dominance and others. Considering these affective words from perspectives of evaluation, interaction and sensory, we extract 16 words (8 pairs) as emotional keywords of VSL, as shown in figure 2.

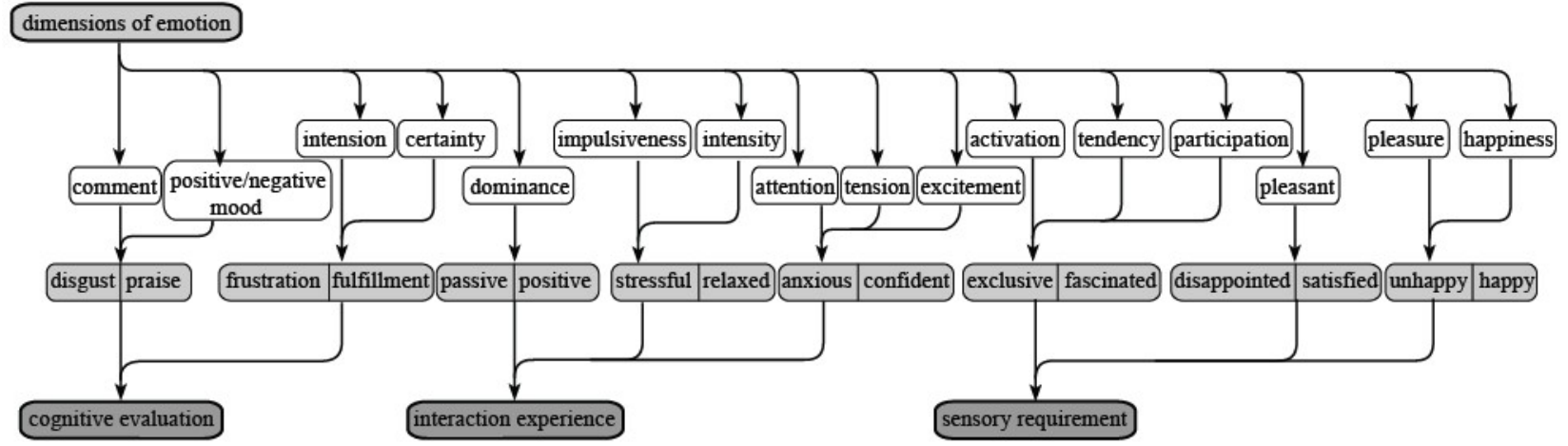

Fig. 2. Emotional Keywords Extraction of VSL based on Affective Design

2 ) Pictographic Picture in VSL design. As a new method of stimulating ideas and providing visual reminders of emotional details especially to children, pictographic pictures were used to measure emotions of learners, see Figure 3. Each picture in (a) is appropriate in one particular situation of (b). In considering that, children have an innate inclination about drawings and pictures, in this case, we develop appropriate frameworks for organising memories, to pick out pictographic pictures with interesting facial expression, which are the key to children to explain the feelings physically and biologically, and get results according to principles. 


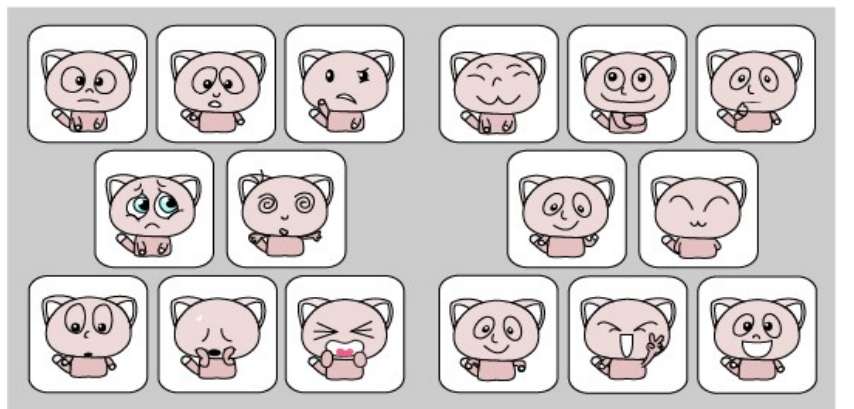

(a)

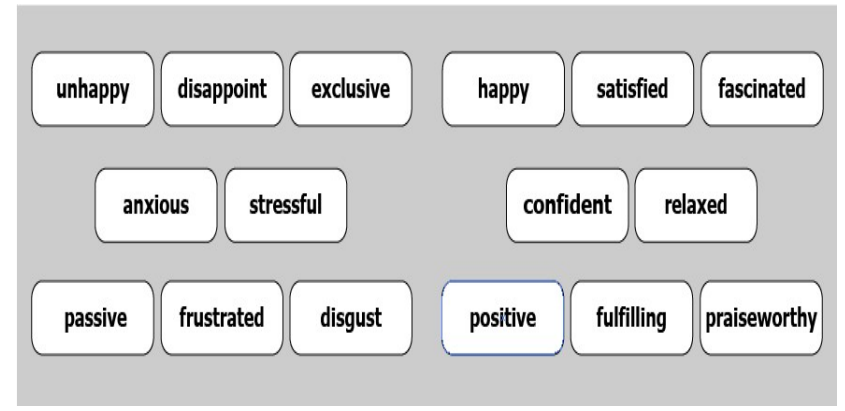

(b)

Fig. 3. Pictographic Pictures of Emotional Elements for VSL

In self-report emotional response to VSL, each pictographic picture contains a 5-layered rating criteria, i.e. strongly agree, highly agree, medium agree, slightly agree, neither agree nor disagree, asking the learner to click the mouse to select, which represent a more valid

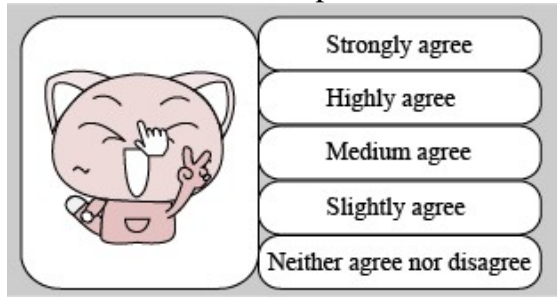

(a)

estimate of the learner's current level of emotional experience, as in Figure 4(a). If chosen, the background of the picture will show the corresponding colour, as in Figure 4(b).

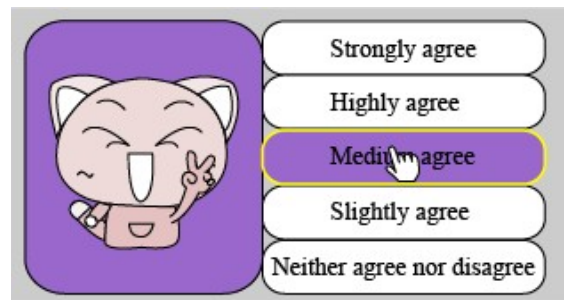

(b)

Fig. 4. Affective Evaluation Model for VSL

\section{DISCUSSION}

From emotional keywords extractions to pictographic pictures to measure learning emotions, the framework stresses the importance of providing learners with an affective learning experience, if learners construct their knowledge through active involvement, it is in part the inner motivations that provides the purposes for learning and continue. As we broaden the conception of learning emotions, not merely the instructional context, the field of VSL design needs to be open not only to the contributions of high-tech learning tools, but also to the potential discoveries of an inquiry into the emotional experience.

\section{REFERENCES}

[1] G. C. Goodwin, A. M. Medioli, W. Sher, L. B. Vlacic and J.S. Welsh, "Emulation-based Virtual Laboratories: A Low-Cost Alternative to Physical Experiments in Control Engineering Education,” IEEE Trans. on Educ.; 2011; 48-55.

[2] S. D. Olabarriaga, T. Glatard and P. T. de Boer, "A virtual laboratory for medical image analysis," IEEE Trans. on Information Tech. in Biomedicine; 2010; 979-985.

[3] Nagamachi M. Kansei Engineering in Consumer Product Design. The Magazine of Human Factors Applications; 2002; 5-9.

[4] Ark W, Dryer D, Lu D. The emotion mouse. Proc. of HCI International on Human-Computer Interaction: Ergonomics and User Interfaces 1999; 823.

[5] Gaulin, Steven J. C. and Donald H. McBurney. Evolutionary Psychology. Prentice Hall; 2003.

[6] Myers, David G. Theories of Emotion. Psychology: 7th Ed, New York: Worth Publishers; 2004.

[7] Liu Y, Tao L, Fu X. The Analysis of PAD Emotional State Model Based on Emotion Pictures. J. of Image and Graphics; 2009; 753-758. 